\title{
Leishmaniose tegumentar americana: flebotomíneos de área de transmissão, no município de Pedro de Toledo, região sul do Estado de São Paulo, Brasil
}

\author{
American tegumentary leishmaniasis: phlebotominae of \\ transmission area in the Pedro deToledo County, in the South \\ region of São Paulo State,Brazil \\ Maria de Fátima Domingos, G lória Cristina Carreri-Bruno, Ricardo Mário de \\ Carvalho Ciaravolo, Eunice Aparecida Bianchi Galati, Dalva Marli Valério \\ Wanderley e Fernando Motta de Azevedo Corrêa
}

\begin{abstract}
Resumo Considerado como área endêmica de leishmaniose tegumentar americana (LTA), o Vale do Ribeira, na região sul do Estado de São Paulo, teve 929 casos notificados nos últimos 15 anos. Com o objetivo de identificar a fauna flebotomínea, foram realizadas capturas quinzenais em área rural do município de Pedro de Toledo, durante um ano, a partir de maio de 1994. Foram utilizadas armadilhas de Shannon no peridomicilio e armadilhas luminosas, tipo CDC, nos ambientes: domiciliar (intra e peri) e florestal (margem e interior), instaladas a partir do crepúsculo vespertino. Foram coletadas 8 espécies de flebotomíneos, totalizando 11.096 exemplares, sendo Lutzomyia intermedia a espécie dominante (96,4\%). Esta espécie mostrou-se mais freqüente na primeira metade da noite, ocorrendo o ano inteiro, preferencialmente no ambiente domiciliar, o que indica sua preferência por ambiente antrópico. Os dados do presente trabalho ratificam $\mathrm{L}$. intermedia como importante espécie vetora de $L T A$ no Vale do Ribeira.
\end{abstract}

Palavras-chaves: Phlebotominae. Fauna flebotomínea. Ecologia de vetores. Leishmaniose tegumentar americana.

Abstract Considered as an American tegumentary leishmaniasis (ATL) endemic area, Ribeira
Valley, in the south region of São Paulo State, presented 929 notified cases in the last 15 years.
Aiming to identify the phlebotomine fauna, captures were performed fortnightly in the rural area
of Pedro de Toledo County during one year, from may 1994 onwards. Set at dusk Shannon traps
were used in the peridomicile and CDC light traps both intra and peridomiciliarly and at the
edge and in the forest. Eight species summing to 11096 specimens were caught, L.intermedia
being the dominant one (96,4\%). More frequent all over the year during the first half of the night
preferably in the domicile surroundings this species clearly indicated its preference for the
anthropic environment. The data presented in this paper ratify L.intermedia as an important ATL
vector species in the Ribeira Valley.

Key-words: Phlebotominae. Phlebotomine fauna. Vector ecology. American tegumentary leishmaniasis.

\footnotetext{
Superintendência de Controle de Endemias (SUCEN) e Faculdade de Saúde Pública da Universidade de São Paulo, São Paulo, SP. Auxílio financeiro:PCDEN e SUCEN

Endereço para correspondência: Maria de Fátima Domingos. Superintendência de Controle de Endemias. Rua João Ramalho 587, 11310-050 São Vicente, SP, Brasil.

Fax: (013) 467-2442.

Recebido para publicação em 17/09/97.
} 
A ocorrência de leishmaniose tegumentar americana (LTA) na região do Vale do Ribeira, sul do Estado de São Paulo, foi assinalada a partir de 1956 com a descrição dos primeiros casos autóctones por Forattini e Oliveira? Atualmente, constitui importante área de transmissão no Estado pois, cerca de $30 \%$ dos casos de LTA notificados, em 1993, tiveram municípios dessa região como local provável de transmissão, segundo dados do Centro de Vigilância Epidemiológica - CVE (Secretaria de Estado da Saúde do Estado de São Paulo).

Nessa região, a doença manifestou-se endemicamente, após décadas de colonização pelo homem, quando não mais existia a floresta primária nos níveis topográficos mais baixos. Não há evidências do padrão clássico de transmissão relacionado com colonização recente e deflorestamento, o que talvez possa ser explicado pela precariedade de condições favoráveis à transmissão de LTA ao homem, na floresta perenifólia higrófila do Sistema da Serra do Mar, sugerindo que a transmissão ocorra no ambiente extraflorestal8 12 .

O conhecimento da fauna flebotomínea e de seu comportamento tem sido objeto de várias investigações. Lutzomyia intermedia, que apresenta comportamento antropofílico, hábito noturno e predomínio no comparecimento ao ambiente domiciliar em relação a outras espécies, vem sendo incriminada como principal vetor4 891011.

Com o objetivo de ampliar o conhecimento sobre a epidemiologia de LTA na região do Vale do Ribeira, desenvolveu-se o presente estudo que visou investigar a distribuição espacial, sazonal e o ritmo nictimeral da fauna flebotomínea, no município de Pedro de Toledo, o qual se destaca na incidência da doença.

\section{MATERIAL E MÉTODOS}

A área de estudo localiza-se em zona rural do município de Pedro de Toledo (241ㅜㅇ de latitude sul e $47^{\circ} 14^{\prime}$ de longitude oeste), região do Vale do Ribeira, sul do Estado de São Paulo. Com uma área de $631 \mathrm{~km}^{2}$ e altitude de $40 \mathrm{~m}$, limita-se com seis outros municípios paulistas: Itariri, Miracatu, Iguape, Itanhaém, Juquitiba e
Peruíbe e dista aproximadamente $150 \mathrm{~km}$ da capital5 (Figura 1).

O clima na área é quente e úmido: Tropical de Altitude, segundo Köppen, com temperaturas médias nos meses mais quentes, superiores a $22^{\circ} \mathrm{C}$ e nos meses mais frios, superiores a $18^{\circ} \mathrm{C}$. A pluviosidade anual é elevada, acima de

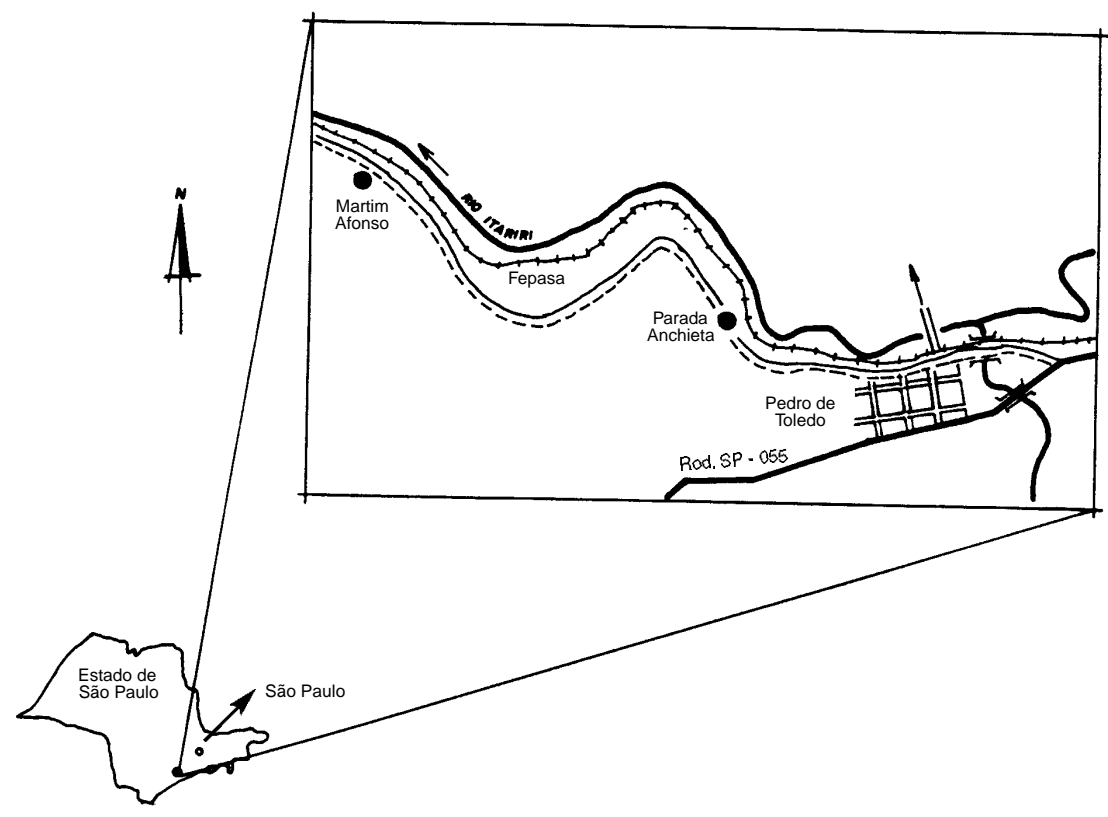

Figura 1 - Localizacão geográfica das área de coleta de flebotomíneos nas localidades Parada Anchieta e Martim Atonso, município de Pedro de Toledo, Estado de São Paulo, SP. 
$1500 \mathrm{~mm}$. A Mata Atlântica (tipo perenifólia higrófila) permanece luxuriante na região e cobre pelo menos $2 / 3$ do município, porém, em alguns pontos, esta cobertura foi quase completamente substituída por pastagens e pela agricultura, com destaque para a bananicultura, base econômica de Pedro de Toledo6 13.

As investigações foram realizadas em duas moradias habitadas, distantes $850 \mathrm{~m}$ uma da outra, situadas nas localidades Parada Anchieta (casa 1) e Martim Afonso (casa 2), localizadas a cerca de $30 \mathrm{~m}$ da mata e, respectivamente, a $5,8 \mathrm{~km}$ e 6,7km da área urbana do município (Figura 1).

Durante um ano, com início em maio de 1994, periodicidade quinzenal e a partir do crepúsculo vespertino, foram efetuadas capturas de flebotomíneos adultos nos dois sítios de coleta, utilizando-se os seguintes procedimentos:

ambiente domiciliar: instalou-se simultâneamente no intradomicílio uma armadilha luminosa tipo CDC operando por 12 horas ininterruptamente e no peridomicílio, da casa 1, em curral e da casa 2, em pocilga, uma armadilha CDC, funcionando durante 12 horas, observados porém dois períodos de 6 horas para troca dos recipientes coletores. Ainda no peridomicílio de cada uma das casas, com armadilhas do tipo Shannon, realizaram-se coletas durante 6 horas, sendo que, a cada 3 meses, com início em agosto, o período foi ampliado para 12 horas. Em cada armadilha de Shannon, empregando tubos mortíferos embebidos em clorofórmio, dois indivíduos realizaram as capturas, sendo os exemplares acondicionados em caixas entomológicas a intervalos regulares de uma hora.

ambiente florestal: foi empregada uma armadilha CDC no interior e outra na margem da mata com a mesma metodologia utilizada no peridomicílio.

As informações sobre a temperatura média mensal foram aquelas registradas para a área

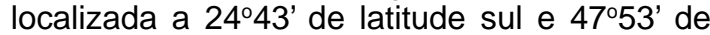
longitude oeste, pela Estação Experimental de Pariquera-Açu do Instituto Agronômico da Secretaria de Estado da Agricultura de São Paulo referentes a Região do Vale do Ribeira. As alturas pluviométricas mensais foram obtidas do Departamento de Águas e Energia Elétrica (DAEE) da Secretaria de Estado de Recursos Hídricos, Saneamento e Obras do Estado de São Paulo. Foram selecionadas as informações referentes ao município de Itariri, por ser o mais próximo a Pedro de Toledo, uma vez que para este município os dados encontravam-se incompletos, impossibilitando a análise no período.

\section{RESULTADOS}

Foram coletadas oito espécies de flebotomíneos: Lutzomyia intermedia (Lutz \& Neiva, 1912), L. fischeri (Pinto, 1926), L. migonei (França,1920), L. ayrozai (Barretto \& Coutinho, 1940), L. pascalei (Coutinho \& Barretto, 1940), L. geniculata (Mangabeira, 1941), L. pestanai (Barretto \& Coutinho, 1941), L. firmatoi (Barretto, Martins \& Pellegrino, 1956), totalizando 11.096 exemplares, sendo 4.173 machos $(37,6 \%)$ e 6.923 fêmeas $(62,4 \%)$. A espécie dominante foi L. intermedia com $96,4 \%$ do total de flebotomíneos coletados (Tabela 1).

Os resultados obtidos com armadilhas CDC, segundo ambiente, apontam o peridomicílio com maior diversidade de fauna flebotomínea, com sete espécies. Quatro delas ocorreram no intradomicílio: $L$. intermedia, $L$. fischeri, $L$. migonei e L. ayrozai (Tabela2). L. intermedia foi a única espécie coletada em todos os

Tabela 1 - Flebotomíneos coletados segundo sexo, nas localidades Parada Anchieta e Martim Afonso, no município de Pedro de Toledo, Estado de São Paulo, maio/1994 a maio/1995.

\begin{tabular}{|c|c|c|c|c|}
\hline Espécies & Macho & Fêmea & Total & $\%$ \\
\hline Lutzomyia intermedia & 4093 & 6607 & 10700 & 96,4 \\
\hline L. fischeri & 6 & 211 & 217 & 2,0 \\
\hline L. migonei & 46 & 57 & 103 & 0,9 \\
\hline L. ayrozai & 16 & 35 & 51 & 0,5 \\
\hline L. pascalei & 10 & 9 & 19 & 0,2 \\
\hline L. pestanai & 1 & 2 & 3 & - \\
\hline L. geniculata & 1 & 1 & 2 & - \\
\hline L. firmatoi & - & 1 & 1 & - \\
\hline Total & 4173 & 6923 & 11096 & 99,9 \\
\hline
\end{tabular}


ambientes, tanto domiciliar como florestal, com maior freqüência no primeiro e predominância no peridomicílio. No ambiente domiciliar houve maior proporção de machos em relação às fêmeas, destacando-se o intradomicílio da casa
1, cuja razão macho/fêmea foi de 3,7 em relação aos demais ambientes que foi cerca de 1,5. Já no ambiente florestal, a mesma foi de cerca de 0,6 (Tabela. 2).

A distribuição horária de L. intermedia, no

Tabela 2 -Flebotomíneos capturados em armadilha CDC segundo espécie, ambiente e sexo, nas localidades Parada Anchieta (casa 1) e Martim Afonso (casa 2), no município de Pedro de Toledo, Estado de São Paulo, maio/1994 a maio/1995.

\begin{tabular}{|c|c|c|c|c|c|c|c|c|c|c|c|c|c|c|c|}
\hline \multirow{4}{*}{ Espécies } & \multicolumn{8}{|c|}{ Ambiente domiciliar } & \multicolumn{4}{|c|}{ Ambiente florestal } & & & \multirow{4}{*}{ Total } \\
\hline & \multicolumn{4}{|c|}{ intra } & \multicolumn{4}{|c|}{ peri } & \multicolumn{2}{|c|}{ margem } & \multicolumn{2}{|c|}{ interior } & \multicolumn{2}{|c|}{ Subtotal } & \\
\hline & \multicolumn{2}{|c|}{ casa 1} & \multicolumn{2}{|c|}{ casa 2} & \multicolumn{2}{|c|}{ casa 1} & \multicolumn{2}{|c|}{ casa 2} & \multirow[b]{2}{*}{$\mathrm{M}$} & \multirow[b]{2}{*}{$\mathrm{F}$} & \multirow[b]{2}{*}{ M } & \multirow[b]{2}{*}{$\mathrm{F}$} & \multirow[b]{2}{*}{$\mathrm{M}$} & \multirow[b]{2}{*}{$\mathrm{F}$} & \\
\hline & $\mathrm{M}$ & $\mathrm{F}$ & $\mathrm{M}$ & $\bar{F}$ & $\mathrm{M}$ & $\mathrm{F}$ & $\mathrm{M}$ & $\mathrm{F}$ & & & & & & & \\
\hline L. intermedia & 285 & 77 & 115 & 70 & 402 & 261 & 1150 & 748 & 61 & 93 & 16 & 24 & 2029 & 1273 & 3302 \\
\hline L. fischeri & - & - & - & 1 & - & 1 & - & 13 & - & 11 & 3 & 13 & 3 & 39 & 42 \\
\hline L. migonei & 1 & - & 1 & 11 & - & 1 & 3 & - & 1 & 4 & - & - & 6 & 16 & 22 \\
\hline L. ayrozai & - & - & - & - & 1 & - & 1 & 3 & 1 & - & 13 & 23 & 16 & 26 & 42 \\
\hline L. pascalei & - & - & - & - & - & - & 2 & 1 & 2 & 1 & 6 & 7 & 10 & 9 & 19 \\
\hline L. geniculata & - & - & - & - & - & - & - & 1 & - & - & - & - & - & 1 & 1 \\
\hline L. firmatoi & - & - & - & - & - & - & - & 1 & - & - & - & - & - & 1 & 1 \\
\hline Total & 286 & 77 & 116 & 82 & 403 & 263 & 1156 & 767 & 65 & 109 & 38 & 67 & 2064 & 1365 & 3429 \\
\hline
\end{tabular}

$\mathrm{M}=$ macho; $\mathrm{F}$ = fêmea,

primeiro período, segundo a média de Williams, mostrou-se diferente entre as 2 casas. Na casa 1, a maior expressividade deu-se na primeira hora, com decréscimo na segunda e manteve-se com valores próximos ao desta até a quinta hora, diminuindo na sexta hora. Já na casa 2, ocorreu aumento gradativo com pico na quinta hora e ligeiro decréscimo na sexta hora (Figura 2).
Considerando somente as quatro capturas de 12 horas verificou-se que a espécie apresentou, nas primeiras horas, na casa 1 comportamento semelhante ao das coletas quinzenais e na casa 2 houve aumento até a $6^{a}$ hora, com pequenas oscilações. Houve, em ambas as casas, declínio acentuado na segunda metade da noite (Figura 3). Nas capturas realizadas com armadilha CDC,

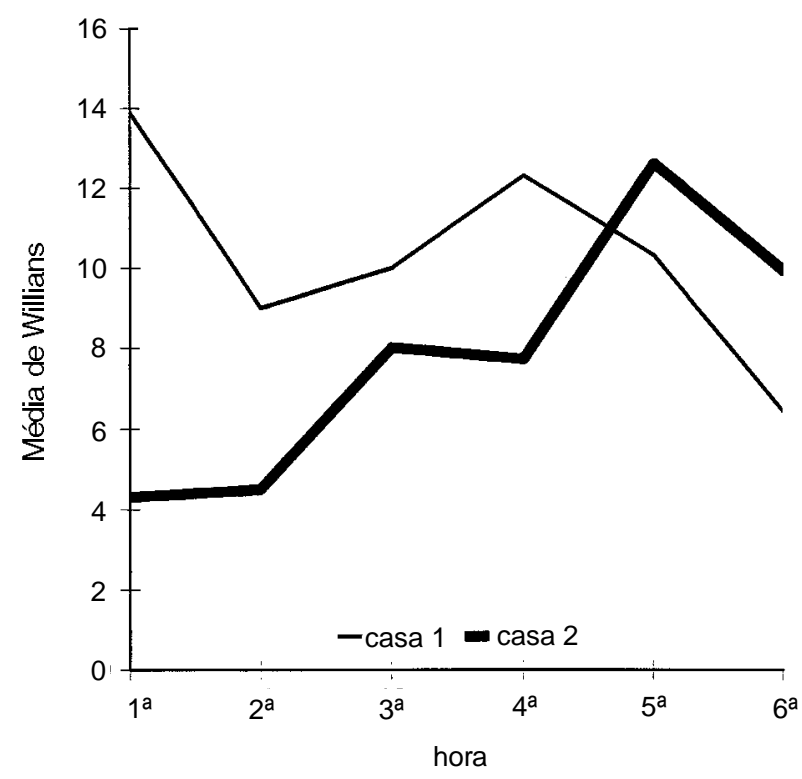

Figura 2 - Freqüência horária de L.intermedia capturada em armadilha de Shannon, com periodicidade quinzenal, nas localidades de Parada Anchieta (casa 1) e Martim Afonso (casa 2), no município de Pedro de Toledo, Estado de São Paulo, maio/1994 a maio/1995. 


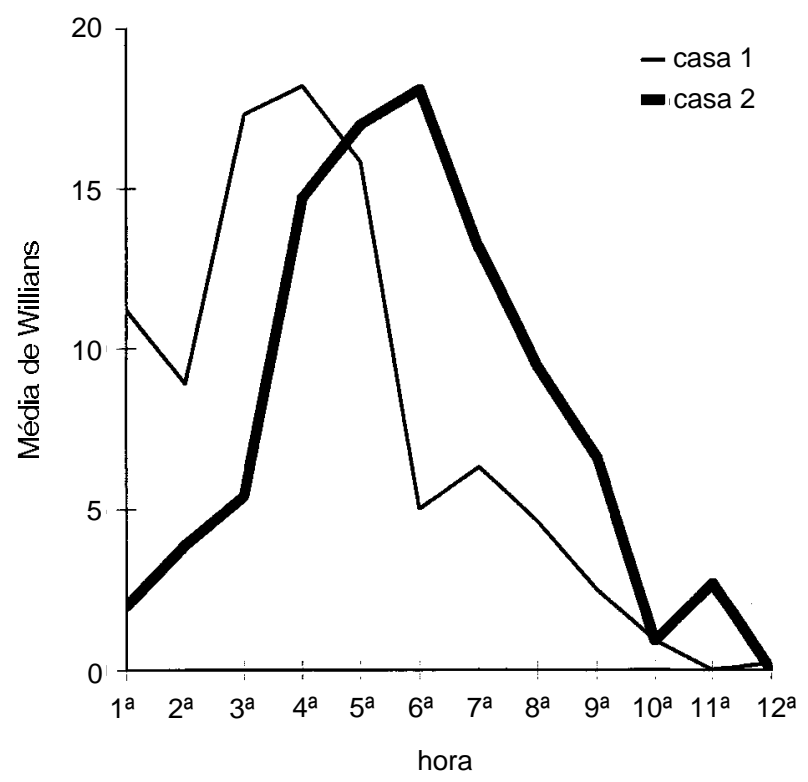

Figura 3 - Freqüência horária de Lutzomyia intermedia capturada em armadilha de Shannon a partir do crepúsculo vespertino, com periodicidade trimestral, nas localidades de Parada Anchieta (casa 1) e Martim Afonso (casa 2), no município de Pedro de Toledo, Estado de São Paulo, maio/1994 a maio/1995.

Tabela 3 - Distribuição de L.intermedia capturada em armadilha CDC segundo período de coleta, ambiente e sexo, nas localidades Parada Anchieta e Martim Afonso, município de Pedro de Toledo, Estado de São Paulo, maio/1994 a maio/1995.

\begin{tabular}{lccccc}
\hline \multirow{2}{*}{ Ambiente } & \multicolumn{2}{c}{$1^{0}$ Período } & & \multicolumn{2}{c}{$2^{0}$ Período } \\
\cline { 2 - 3 } \cline { 5 - 6 } & macho & fêmea & & macho & fêmea \\
\hline Peridomicílio & 1190 & 742 & & 362 & 267 \\
Margem da mata & 44 & 53 & & 17 & 40 \\
Interior da mata & 14 & 22 & 2 & 2 \\
\hline Total & 1248 & 817 & 381 & 309 \\
\hline
\end{tabular}

também foi verificada maior atividade desta espécie no primeiro perlodo da noite (Tabela 3).

A freqüência mensal de L. intermedia capturada em armadilha de Shannon, segundo a média de Williams, foi distinta nas duas casas. $\mathrm{Na}$ casa 1 mostrou-se freqüente no inverno com o maior pico no mês de agosto. Na casa 2, pôde-se observar uma tendência de ciclicidade bimensal, porém, assinalou-se um pico em janeiro (Figura 4). A Figura 5 apresenta distribuição mensal das alturas de chuva e temperaturas médias.

\section{DISCUSSÃO}

Das oito espécies identificadas neste estudo (Tabela 1), somente $L$. firmatoi foi assinalada pela primeira vez na região. L. pestanai foi citada como L. shannoni (Dyar, 1929) por Gomes e 


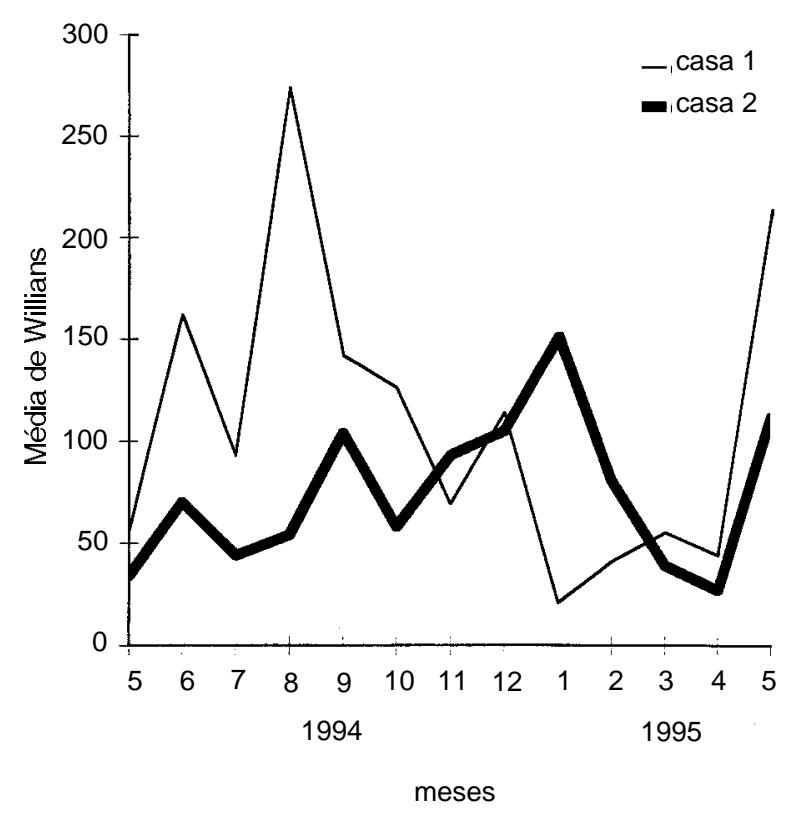

Figura 4 - Freqüência mensal de Lutzomyia intermedia capturada em armadilha de Shannon, no primeiro período, nas localidades de Parada Anchieta (casa 1) e Martim Afonso (casa 2), no município de Pedro de Toledo, Estado de São Paulo, maio/1994 a maio/1995.

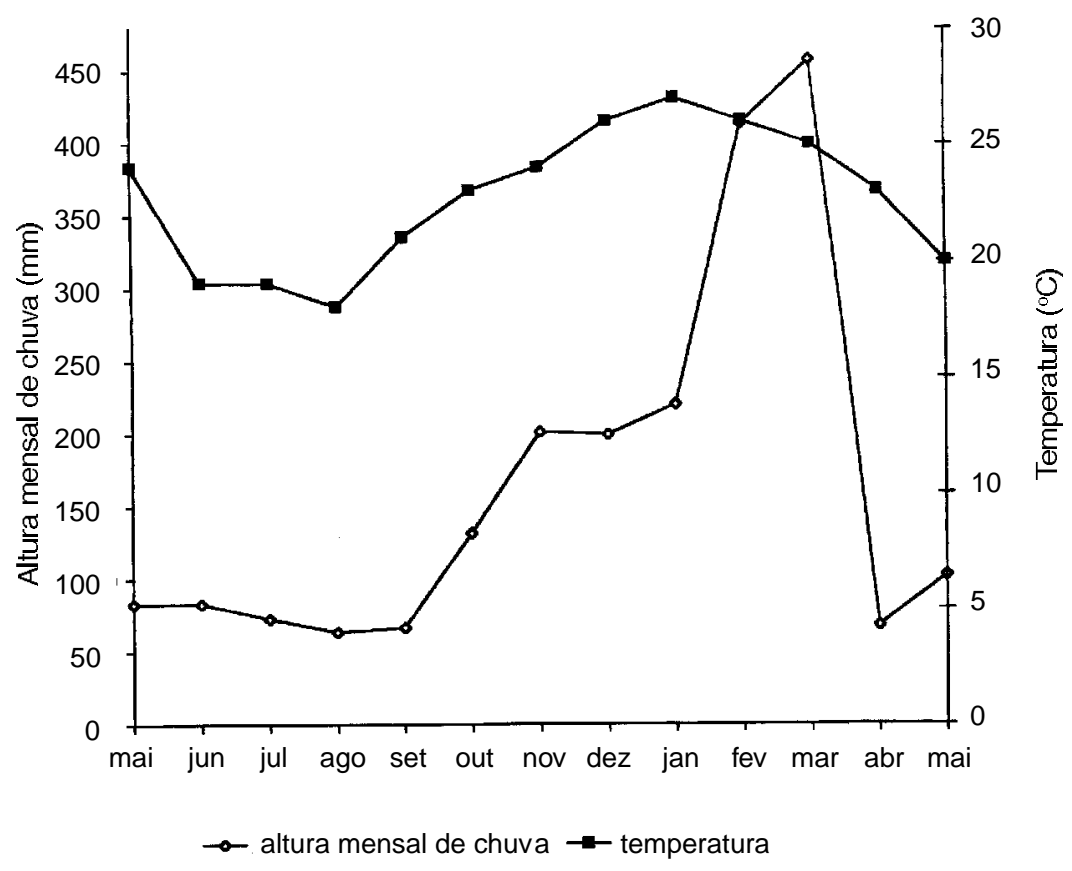

Figura 5 - Distribuição das alturas de chuva $(\mathrm{mm})$ no município de Itariri e temperatura média mensal para a região do Vale do Ribeira, localizada entre os paralelos 2443'S e 47053'W. Estado de São Paulo, maio/1994 a maio/1995. 
Galati11 (informações pessoais de um dos colaboradores E.A.B.G.), sendo que tal equívoco deu-se em função das fêmeas de ambas espécies serem muito próximas, e machos, que são facilmente distinguíveis, não terem sido coletados, embora, erroneamente, constem da Tabela 1 do referido artigo. L. geniculata vem sendo assinalada para a área como L. guyanensis (Floch \& Abonnenc, 1941) pois Forattini 3 a considera como sinônimo dessa espécie, sendo a mesma restrita à Guiana Francesa, segundo Young \& Duncan 199414.

Foi nítida a predominância de $L$. intermedia tanto no ambiente domiciliar $(98,7 \%)$ como no florestal em relação às demais espécies (Tabelas 1 e 2) com maior densidade no primeiro, tal como observado por Forattini et al ${ }^{4}$. Essa elevada densidade em ambiente modificado, no qual se inclui o domiciliar, tem contribuído para incriminá-la como vetor da LTA 19.

A maior proporção de machos de $L$. intermedia nas coletas domiciliares com armadilhas tipo CDC (Tabela 2) pode ser interpretada como uma necessidade biológica essencial de assegurar a fecundação das fêmeas que seriam cortejadas durante seus deslocamentos 19 .

No intradomicílio da casa 1 a razão macho/fêmea foi maior que o dobro da dos demais ambientes domiciliares, isto parece indicar a presença de criadouros da espécie próximos ao domicílio, provavelmente associados a área de escoamento de dejetos da pocilga. Reforça este ponto de vista a freqüência muito mais elevada para ambos os sexos na primeira hora de coleta na casa 1 , em relação aos horários seguintes. Na casa 2, a espécie teve comportamento oposto ao da casa 1 , o que parece indicar que os criadouros encontravamse mais distantes (Figura 2).

Tendo em vista o maior rendimento de $L$. intermedia nas coletas com armadilhas de Shannon no peridomicílio da casa 1 , seria de se esperar que o mesmo se desse com o da CDC instalada neste ambiente. Todavia, tal fato não ocorreu, provavelmente devido a mesma ter sido instalada atrás de uma parede de alvenaria da pocilga, que formava efetiva barreira à atração luminosa da armadilha, ao se considerar que os criadouros estivessem na direção do escoamento dos dejetos da pocilga.

A exemplo do anteriormente observado por
Forattini et $\mathrm{al}^{4}$, tanto nas coletas com CDC como na armadilha de Shannon L. intermedia revelou maior atividade na primeira metade da noite (Tabela 3 e Figura 3).

L. intermedia esteve presente durante $\mathrm{o}$ ano inteiro de forma irregular, assim como observado por Gomes et al9, e apresentou picos no verão e inverno, também apontados por Forattini3 (Figura 4).

Durante e após períodos intensos de precipitação pluviométrica a população de $L$. intermedia tende a reduzir sensivelmente como se pôde depreender pelos resultados obtidos nas coletas de março e abril (Figuras 4 e 5), possívelmente devido ao impacto que as chuvas ocasionam nos criadouros da espécie, os quais parecem estar associados ao solo sob dossel compacto da mata.

A dominância de L. intermedia no ambiente domiciliar, sua atividade durante todo o período noturno e presença contínua ao longo do ano, ratificam esta espécie como vetora de LTA na região do Vale do Ribeira.

\section{AGRADECIMENTO}

A Gerson Laurindo Barbosa pelo auxílio na elaboração do banco de dados do presente estudo.

\section{REFERÊNCIAS BIBLIOGRÁFICAS}

1. Forattini OP. Nota sôbre criadouros naturais de flebótomos em dependências peri-domiciliares, no Estado de São Paulo. Arquivos da Faculdade de Higiene e Saúde Pública 7:157-167, 1953.

2. Forattini OP, Oliveira O. Um foco de leishmaniose tegumentar na zona sul do Estado de São Paulo, Brasil. Arquivos da Faculdade de Higiene e Saúde Pública 11:23-34, 1957.

3. Forattini OP. Entomologia médica.São Paulo, Edgar Blücher/Edusp, v. 4. 1973.

4. Forattini OP, Rabello EX, Serra OP, Galati EAB, Barata JMS. Observações sobre a transmissão da leishmaniose tegumentar no Estado de São Paulo, Brasil.Revista de Saúde Pública 10:31-43, 1976.

5. Fundação Instituto Brasileiro de Geografia e Estatística. Censo demográfico e dados distritais. IX Recenseamento geral do Brasil, 1980. Rio de Janeiro, vol. 1, tomo 1, no 1, 1982.

6. Fundação Instituto Brasileiro de Geografia e Estatística. Anuário Estatístico do Brasil, 1985. Rio de Janeiro, 1986. 
7. Gomes AC, Galati EAB, . Aspectos ecológicos da leishmaniose tegumentar americana.5.Estratificação de atividade espacial e estacional de Phlebotominae (Diptera, Psychodidae) em áreas de cultura agrícola da região do Vale do Ribeira, Estado de São Paulo, Brasil. Memórias do Instituto Oswaldo Cruz 82:467473, 1987.

8. Gomes AC, Galati EAB. Aspectos ecológicos da leishmaniose tegumentar americana. 7. Capacidade vetorial flebotomínea em ambiente florestal primário do sistema da Serra do Mar, região do Vale do Ribeira, Estado de São Paulo, Brasil. Revista de Saúde Pública 23:136-142, 1989.

9. Gomes AC, Rabello EX, Santos JLF, Galati EAB. Aspectos ecológicos da leishmaniose americana. 1 Estudo experimental da freqüência de flebotomíneos a ecótopos artificiais com referência especial a Psychodopygus intermedius. Revista de Saúde Pública 14:540-556, 1980.

10. Gomes AC, Rabello EX, Santos JLF, Galati EAB. Aspectos ecológicos da leishmaniose tegumentar americana. 2. Ecótopo artificial como abrigo de
Psychodopygus intermedius e observações sobre alimentação e reprodução sob influência de fatores físicos naturais. Revista de Saúde Pública 16:149159, 1982.

11. Gomes AC, Santos JLF, Galati EAB. Ecological aspects of America cutaneos leishmaniasis. 4. Observations on the endophilic behavior of the sandfly and vectorial role of Psychodopygus intermedius in the Ribeira Valey region of the S. Paulo State, Brazil. Revista de Saúde Pública 20:280-287, 1986.

12. Gomes AC, Yamamoto YI, Capinzaiki AN, Amaral NMM, Guimarães AJG. Aspectos ecológicos da leishmaniose tegumentar americana. 9. Prevalência incidência da infecção humana nos municípios de Pedro de Toledo e Miracatu, São Paulo, Brasil. Revista do Instituto de Medicina Tropical de São Paulo 34:149-158, 1992.

13. Instituto Brasileiro de Geografia e Estatística. Superintendência de Cartografia.Cartas do Brasil. $1^{\text {a }}$ edição, 1973.

14. Young DG, Duncan MA. Guide to the identification 